\title{
Comparative study of solar cycles 22 to 24 in relation to solar output variability
}

\author{
Sham Singh, Divya Shrivastava, Shri Ram Lahauriya, Adya Prasad Mishra
}

Department of Physics, Awadhesh Pratap Singh University, Rewa, India; shamrathore@yahoo.com, apm_apsu@yahoo.co.in

Received 25 January 2012; revised 27 February 2012; accepted 10 March 2012

\begin{abstract}
The rate of increase in solar activity at the initiation of solar cycle 22 had led the activity during the solar maximum years 1990-1991, be the most severe of any period during the space age. Solar cycle 23 started in May 1996 and ended in December 2008. Total time period of this solar cycle is $\mathbf{1 2 . 6}$ years. The maximum smoothed sunspot number observed during the solar cycle was 120.8 and minimum was 1.7. It is apparent that solar cycle 23 is a long one. Solar cycle 24 (initiated in December 2008) seems to be weak, which will have a slow ramp up, much slower than the late $19^{\text {th }}$ century cycles used for comparison. In the present work, we have considered Sunspot number (Rz) as an index of solar activity, which shows a predominant 11 year cycle. The strength of the solar cycle makes a huge difference to satellite operators. Each solar peak heats and expands the outer atmosphere. The strength of the solar-cycle length, annual average and temperature correlation enables solar cycle length to be used as a climate predictor tool.
\end{abstract}

Keywords: Solar Activity; Cosmic Ray Intensity; Geomagnetic Activity

\section{INTRODUCTION}

Analyses of solar and geomagnetic activity are important to various technologies, including the operation of low-Earth orbiting satellites, electric power transmission grids, geophysical operation, and high frequency radio communications. Influence of solar activity during different phases of solar cycle on geomagnetic disturbance and geomagnetic hazards has been discussed in the literature [1-3]. The entire structure of the Sun's magnetic field changes in 11-year cycle. During a solar cycle the strongest magnetic fields slowly migrate towards the Sun's equator from locations about midway to the Sun's poles. The 11-year period is not constant but varies from one cycle to other between 9.5 to 12.5 years [4]. The position of the sunspots is not random [5]. They first appear in the middle latitudes above and below the Sun's equator. After 11-years when the most cycle begins, the magnetic field poles are reversed. Solar cycles 22 and 23 was 9.7 and 12.6 years long respectively [6,7]. Cycles 22 and 23 both showed large annual average of geomagne tic activity in comparison with most cycle in the record of aa indices. The dramatic variability from one cycle to the next in these sunspot and geomagnetic records shows the difficulty in making empirical predictions of both types of activity. In the present work, we have analyzed the solar output variability during solar cycles 22 to 24 .

\section{SELECTION CRITERIA AND DATA SOURCE}

In the present study, we have studied correlation of Cosmic Ray Intensity with solar activity and geomagnetic activity. The relationship between solar activity and Cosmic Ray Intensity (CRI) in solar cycles 22 to 24 has also been analysed. The significance of the geomagnetic Aa index in tracking long-term solar activity has been documented in the literature [8]. For this purpose we have selected CRI data of Moscow Neutron Monitor (cut off rigidity $2.42 \mathrm{GV}$ ) and the data of geomagnetic activeity (aa Indices and Ap Indices) have been taken from web site (www.geomag.bgs.ac.uk/daaservice/dat). The international SSN data was downloaded from the National Geophysical Data Centre web site

(htt://www.ngdc.noaa.gov/stp/SOLAR/ftpsunspotnumber .html).

\section{RELATIONSHIP BETWEEN SOLAR ACTIVITY AND COSMIC RAY INTENSITY}

To asses level the of correlation between sunspot number and cosmic ray intensity for the solar cycles 22 to 24 , the correlation coefficient between the monthly mean values of these two parameters has been derived. During solar cycle 22 the maximum SSN observed was 
200.3 on August 1990 and minimum 1.1 on June 1987. In cycle 23, maximum SSN was 150.7 on Sept. 2001 and minimum 0.9 on Oct. 2007. Cycle 22 was started in Sept. 1986 after four years it reached maximum level but cycle 23 started in May 1996 and becomes maximum in Sept. 2001, it took more time to reach maxima as compared to cycle 22. The weaker and longer solar cycles of the late $19^{\text {th }}$ and early $20^{\text {th }}$ century were associated with weather that was much colder than that of the late $20^{\text {th }}$ century [9]. Brown and Williams [10] have noticed an extremely high correlation between geomagnetic activity near solar minimum and the size of the next solar cycle. Strong correlations were found between the number of geomagnetic abnormal quite days and the size of the next solar cycle. The relationship of SSN and CRI is clearly seen in Figure 1(a). It is observed from Figure 1(b) that the anti-correlation between SSN and CRI has been observed to $-0.85,-0.57$ and -0.60 during ascending phases of cycles 22, 23 and 24 respectively. The period of minimum CRI is much broader in cycle 23. Cosmic Ray

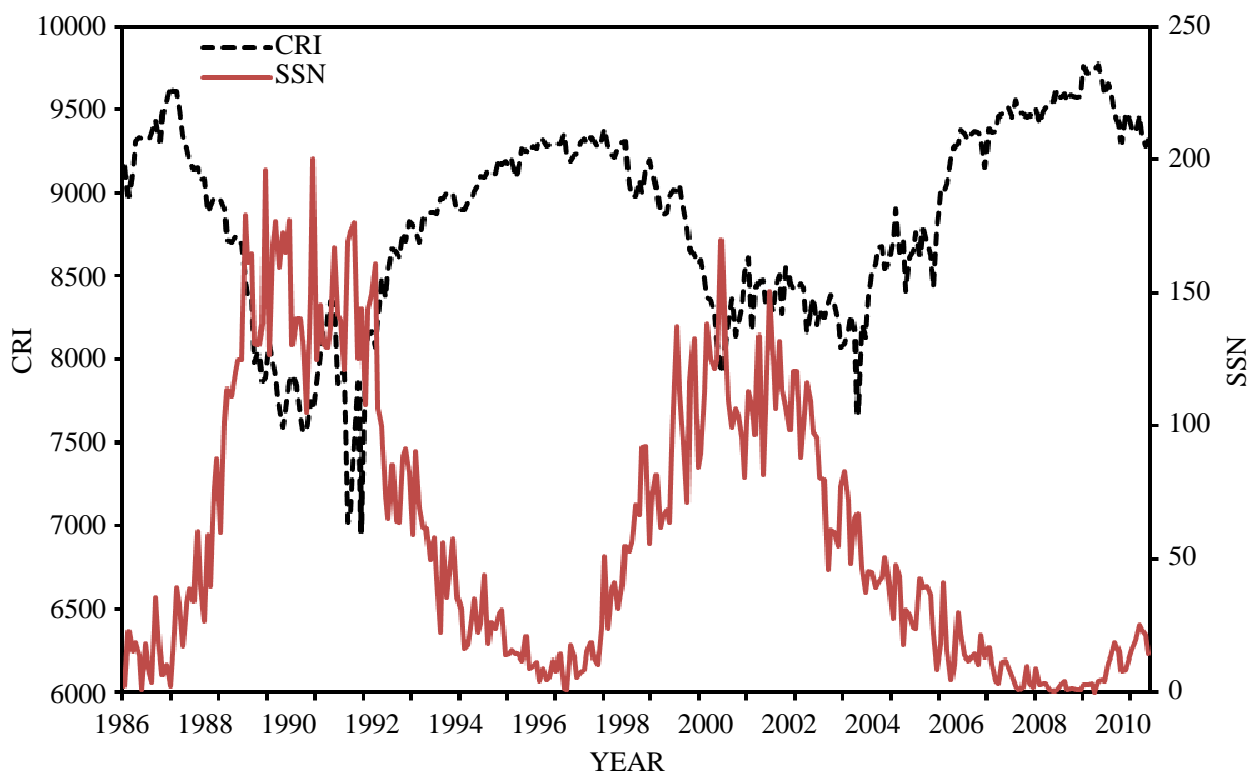

(a)

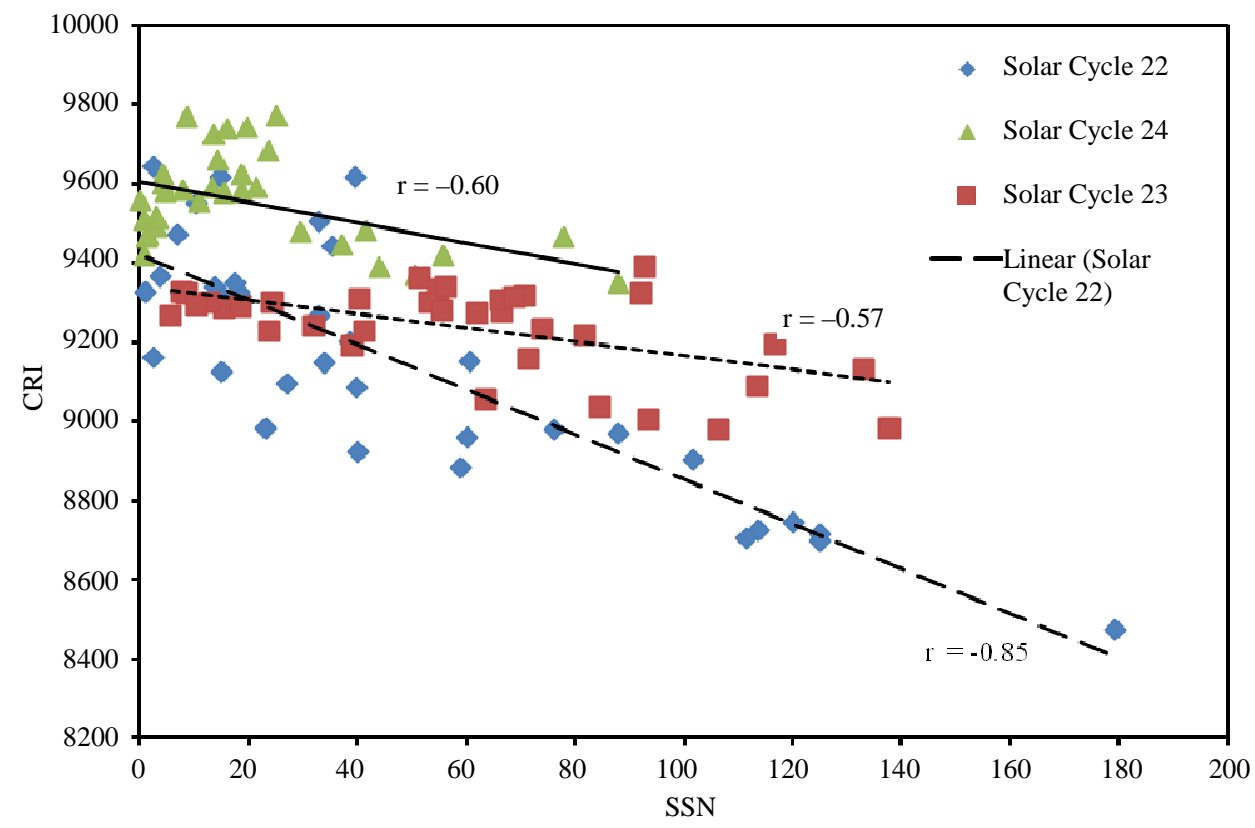

(b)

Figure 1. (a) Shows long term variation of between SSN and CRI for the solar cycles 22 to 24; (b) Shows the cross plot between SSN and CRI for the cycles 22 to 24. 


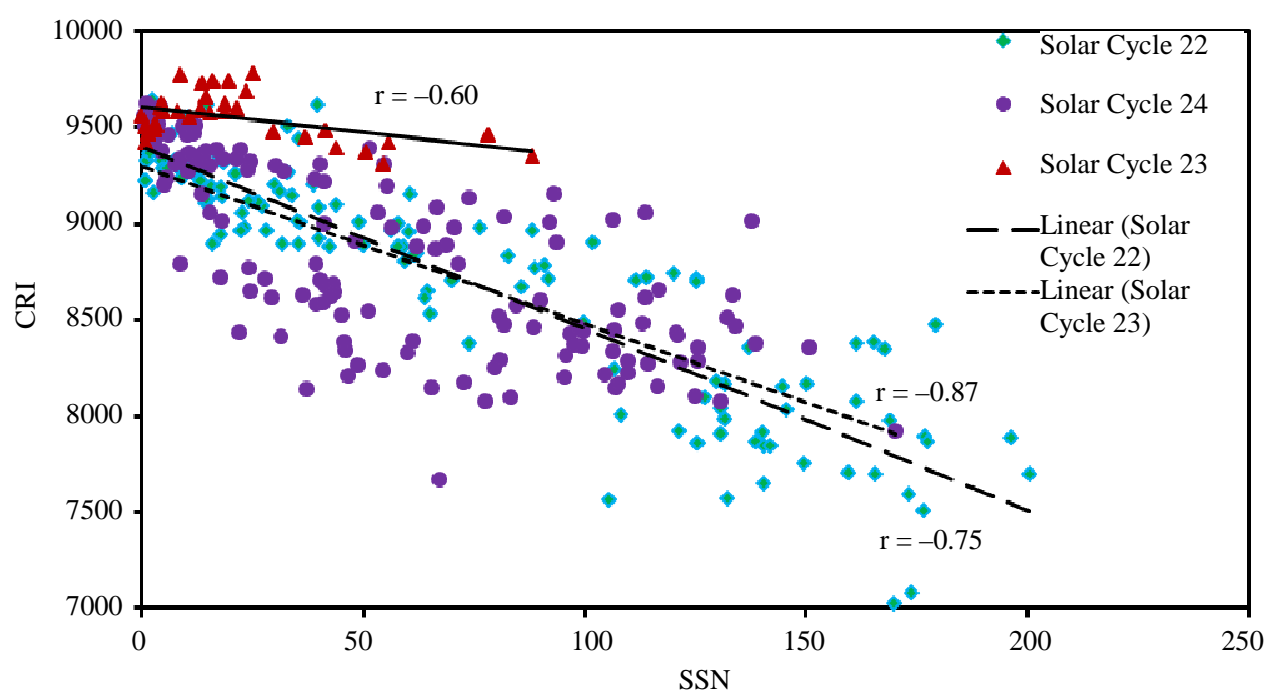

(a)

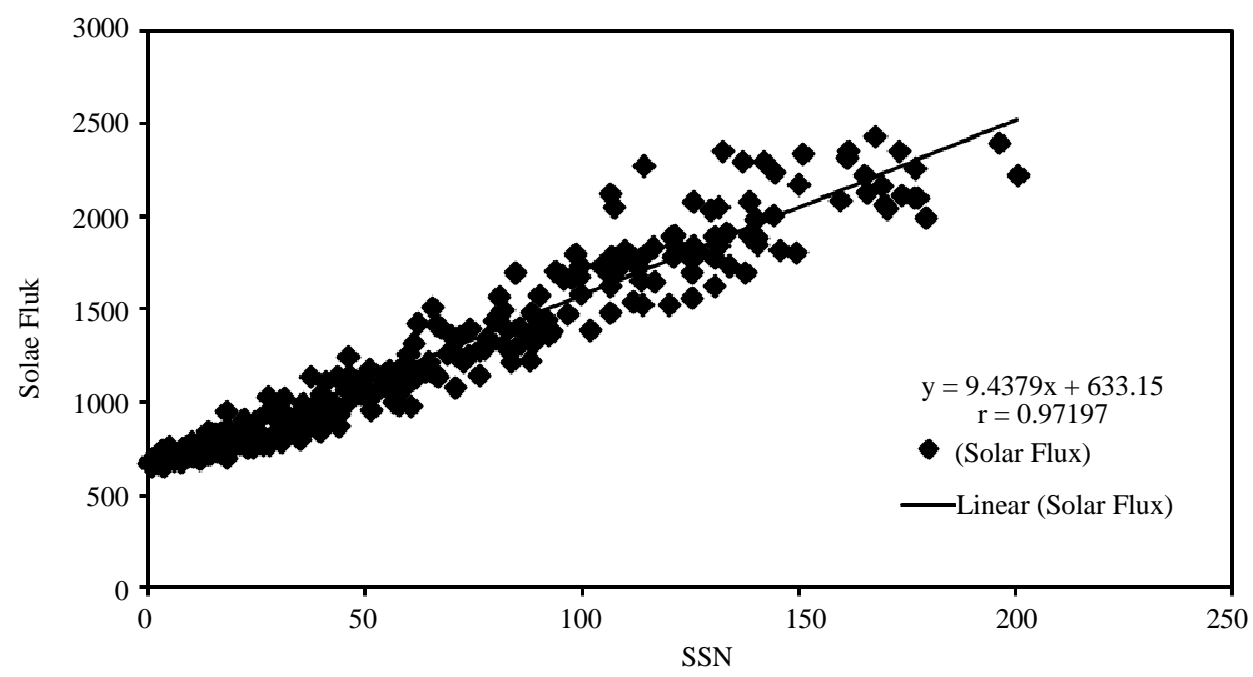

(b)

Figure 2. (a) Shows the cross plot between CRI and SSN for the cycles 22 to 24; (b) Shows the cross plot between Solar Flux and SSN for the cycles 22 to 24.

Intensity is maximum in 1986-1987, after 11 years it occurred in 1996-1997 and again after 11 year in 20062007. The next maximum is expected in 2016-2017. The longterm variation has shown strong negative correlation for cycles 22, 23 and 24. The correlation coefficient between CRI and SSN for the cycles 22, 23 and 24 found to be $-0.87,-0.75$ and -0.60 respectively (Figure $2(\mathbf{a})$ ). We have also obtained the correlation coefficient between Solar Flux and SSN, and positive correlation is found as depicted in (Figure 2(b)).

\section{RELATIONSHIP BETWEEN SOLAR ACTIVITY, CRI AND GEOMAGNETIC ACTIVITY}

We have considered the Ap and aa Indices to observe the long term variations of geomagnetic field disturbances. It is well known that the geomagnetic disturbances are associated with solar outputs emanates from the surface of the Sun, which in turn propagate their energy through solar wind and interplanetary magnetic field to long distance in heliosphere. We have plotted the monthly mean value of SSN, SF, and CRI with monthly mean values of Ap and aa Indices. We have found the positive correlation (correlation coefficient 0.45) between SSN and Ap (Figure 3(a)) for the period 1986 to 2011, which cover the solar cycles 22 to 24. Similar plot is drawn for SSN and aa indices (Figure 3(b)) for which the correlation coefficient is 0.42 and similar result for correlation between SF with Ap \& aa indices (Figures 3(c) and (d)). We have also studied the correlation of 


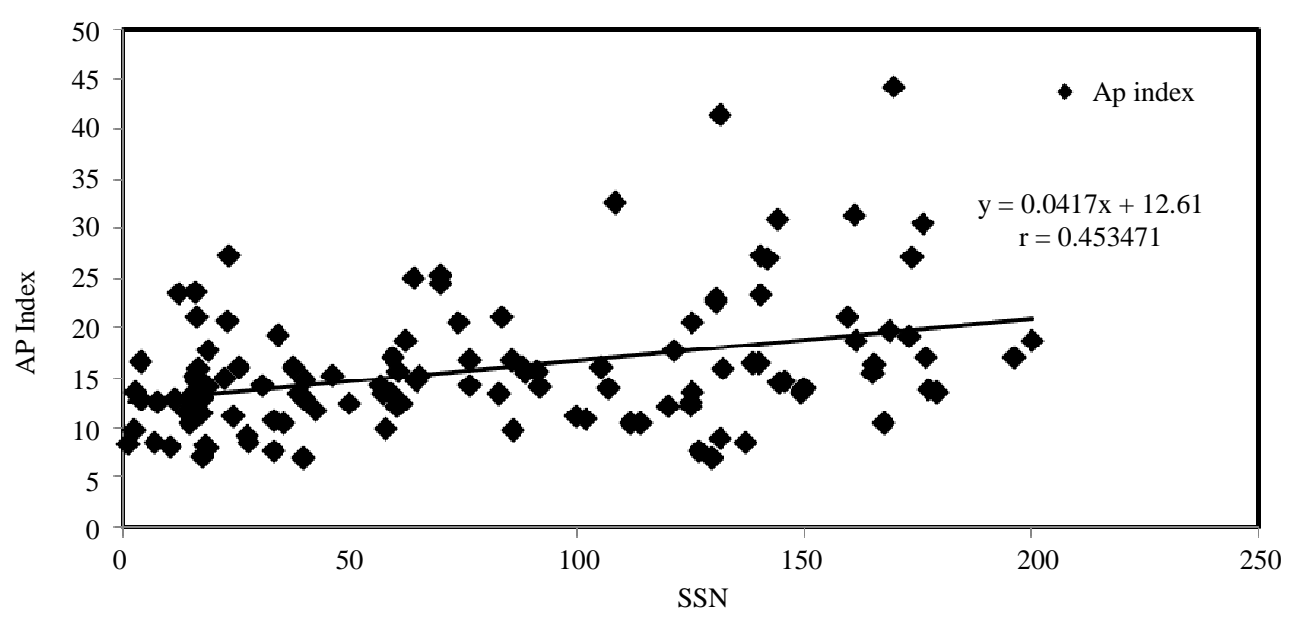

(a)

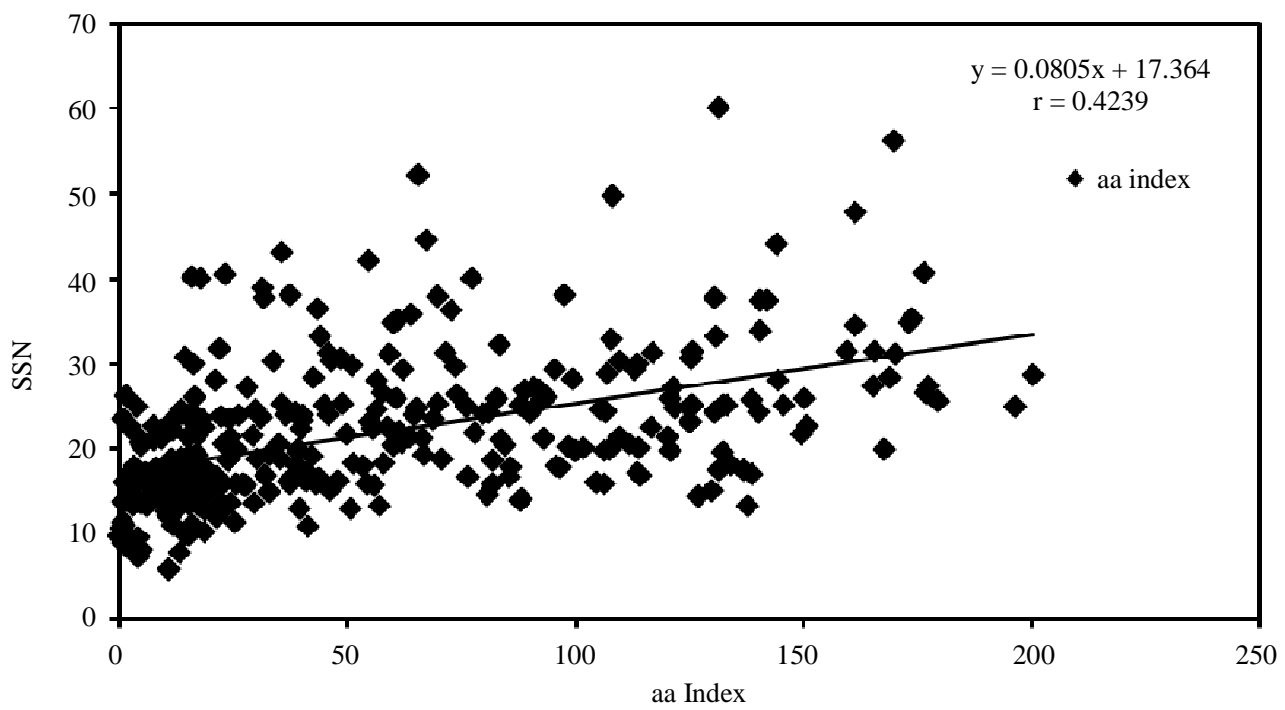

(b)

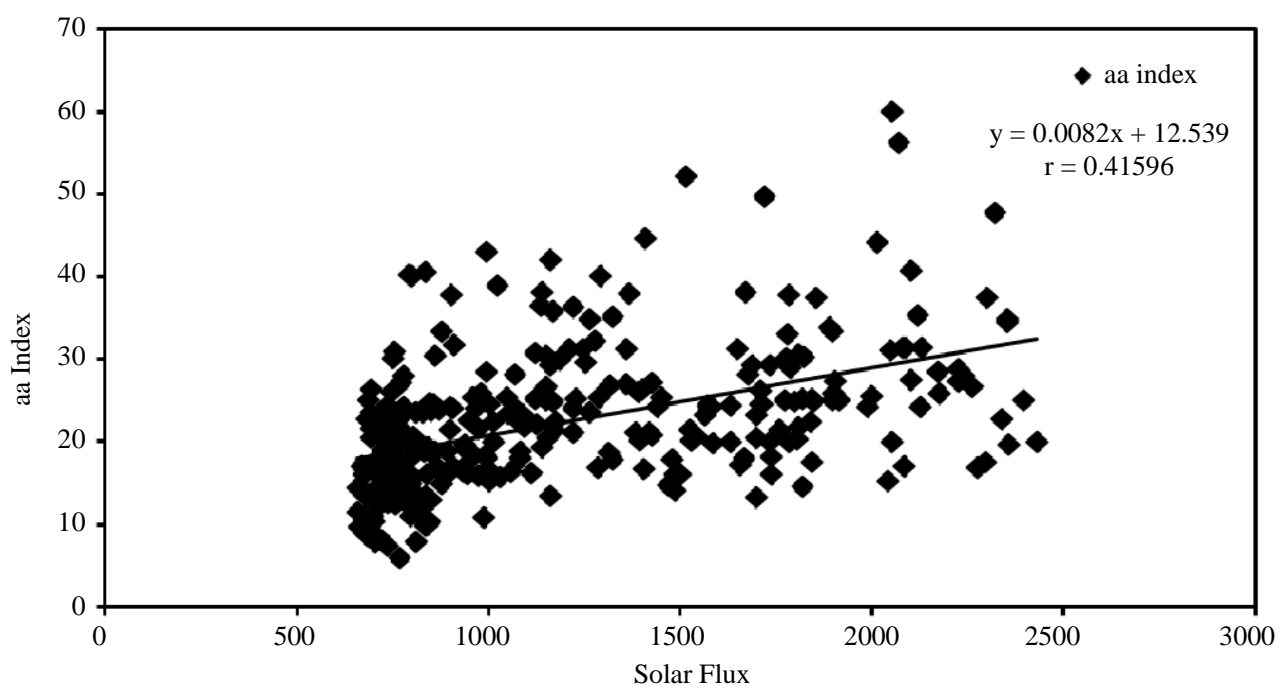

(c) 


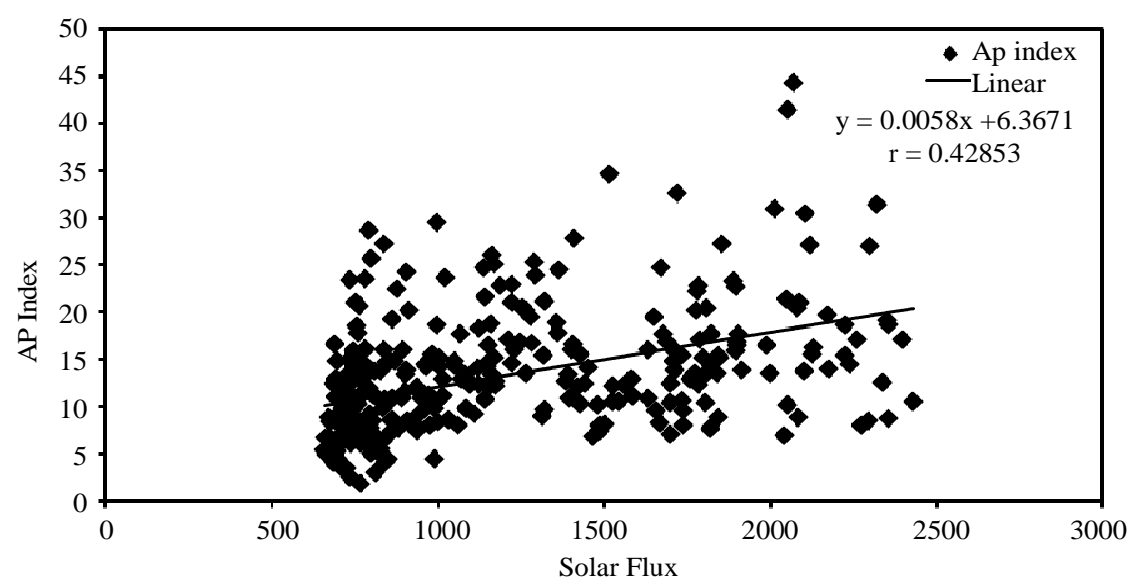

(d)

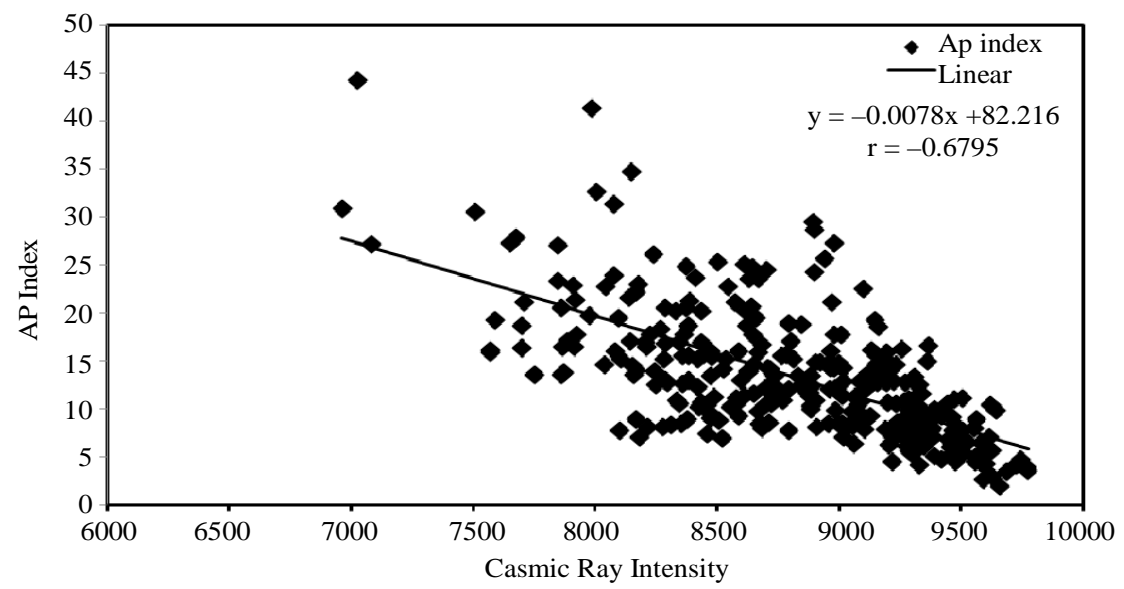

(e)

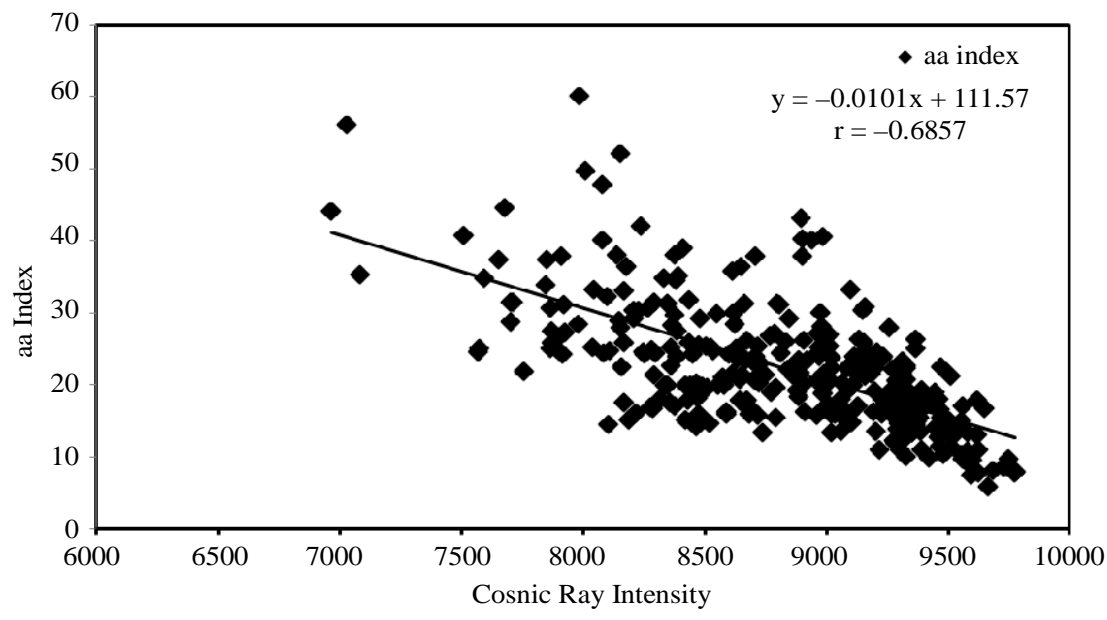

(f)

Figure 3. (a) Shows the cross plot between Ap Index and SSN for the solar cycles 22 to 24; (b) Shows the cross plot between SSN and aa Index for the solar cycles 22 to 24; (c) Shows the cross plot between aa Index and Solar Flux for the cycles 22 to 24; (d) Shows the linear plot between Solar Flux and Ap Index for the cycles 22 to 24; (e) Shows the cross plot between Ap Index and Cosmic Ray Intensity for the cycles 22 to 24; (f) Shows the cross plot between aa Index and Cosmic Ray Intensity for the solar cycles 22 to 24 . 
Table 1. Correlation coefficient between solar activity, cosmic ray intensity and geomagnetic activity for cycle 22 .

\begin{tabular}{lc}
\hline Solar Activity \& Geomagnetic Activity & Correlation Coefficient \\
\hline Sunspot Number \& Solar Flux & 0.98 \\
Sunspot Number \& CRI & -0.87 \\
Solar Flux \& CRI & -0.87 \\
Solar Flux \& Ap Index & 0.45 \\
Solar Flux \& aa Index & 0.39 \\
Sunspot Number \& Ap Index & 0.38 \\
Sunspot Number \& and aa Index & 0.42 \\
CRI \& Ap Index & -0.57 \\
CRI \& aa Index & -0.53 \\
\hline
\end{tabular}

Table 2. Correlation coefficient between solar activity, cosmic ray intensity and geomagnetic activity for cycle 23 .

\begin{tabular}{lc}
\hline Solar Activity \& Geomagnetic Activity & Correlation Coefficient \\
\hline Sunspot Number \& Solar Flux & 0.97 \\
Sunspot Number \& CRI & -0.75 \\
Solar Flux \& CRI & -0.76 \\
Solar Flux \& Ap Index & 0.43 \\
Solar Flux \& aa Index & 0.42 \\
Sunspot Number \& Ap Index & 0.40 \\
Sunspot Number \& and aa Index & 0.42 \\
CRI \& Ap Index & -0.68 \\
CRI \& aa Index & -0.69 \\
\hline
\end{tabular}

Table 3. Correlation coefficient between solar activity, cosmic ray intensity and geomagnetic activity for cycle 24 .

\begin{tabular}{lc}
\hline Solar Activity \& Geomagnetic Activity & Correlation Coefficient \\
\hline Sunspot Number \& Solar Flux & 0.98 \\
Sunspot Number \& CRI & -0.60 \\
Solar Flux \& CRI & -0.59 \\
Solar Flux \& Ap Index & 0.45 \\
Solar Flux \& aa Index & 0.40 \\
Sunspot Number \& Ap Index & 0.47 \\
Sunspot Number \& and aa Index & 0.42 \\
CRI \& Ap Index & -0.52 \\
CRI \& aa Index & -0.42 \\
\hline
\end{tabular}

CRI with Ap and aa indices. The correlation coefficient $-0.67 \&-0.68$ is found as depicted in Figures 3(e) and 3(f)). The correlation coefficient, cosmic ray intensity and geo- magnetic activity for solar cycles 22, 23 and 24 has been shown in Tables 1-3.

\section{REFERENCES}

[1] Dubey, S.C. and Mishra, A.P. (2000) Solar wind streams and intense geomagnetic storms observed during 19861991. Earth Moon and Planets (Netherlands), 84, 23-31. doi:10.1023/A:1026566510077

[2] Dubey, S.C. and Mishra, A.P. (2001) Study of the solar wind streams associated with intense geomagnetic storms. Bulletin of the Astronomical Society of India, 29, 127134.

[3] Dubey, S.C. and Mishra, A.P. (2000) Characteristics of large geomagnetic storms observed during solar cycle 22. Indian Journal of Radio and Space Physics, 29, 51.

[4] Mursala, K. and Ulich, T. (1998) A new method to determine the solar cycle length. Geophysical Research Letters, 25, 1837-1840. doi:10.1029/98GL51317

[5] Lean, J., Skumanich, A. and White, O. (1992) Estimating the Sun's radiative output during the maunder minimum. Geophysical Research Letters, 19, 1591-1594. doi:10.1029/92GL01578

[6] Kane, R.P. (2002) Some implications using the group sunspot number reconstruction. Solar Physics, 205, 383401.

[7] The Sun. Did you say the Sun has spot? Space Today Online.

http://www.spacetoday.org/SolSys/Sun/Sunspots.html. Retrieved 12 August 2010

[8] Feynman, J. and Gu, X.Y. (1986) Prediction of geomagnetic activity on time scales of one to ten years. Reviews of Geophysics, 24, 650-666. doi:10.1029/RG024i003p00650

[9] Loehle, C. (2007) A 2000-year global temperature reconstruction based on non-treering proxies. Energy and Environment, 18, 1049-1058. doi:10.1260/095830507782616797

[10] Brown, G.M. and Williams, W.R. (1969) Some properties of the day-to-day variability of $\mathrm{Sq}(\mathrm{H})$. Planetary and Space Science, 17, 455-469. doi:10.1016/0032-0633(69)90076-2 